\title{
Conhecimentos e Condutas dos Profissionais de Saúde Sobre a Violência Contra a Mulher
}

\author{
Knowledge and Conducts of Health Professionals About the Violence Against Women \\ Conocimiento y Conductas de los Profesionales de la Salud Sobre la Violencia Contra la \\ Mujer
}

\author{
Rafaela Serrano Fusquine ${ }^{1}$ \\ Yasmin Alves de Souza \\ Aucely Corrêa Fernandes Chagas \\ Universidade Católica Dom Bosco (UCDB)
}

\begin{abstract}
Resumo
Introdução: A violência contra a mulher gera um aumento na demanda dos serviços de saúde que são fundamentais no reconhecimento das situações de violência por meio da análise clínica e acolhimento. Objetivo: analisar o conhecimento dos profissionais de saúde da atenção básica diante da violência contra a mulher e verificar suas condutas durante o atendimento. Método: Estudo quantitativo transversal, desenvolvido com trinta profissionais de saúde de uma unidade básica de saúde, por meio de um questionário composto por perguntas de dados pessoais, profissionais e entendimento sobre a violência doméstica. Resultados e discussão: A maioria dos entrevistados nega conhecimento prévio sobre violência doméstica, interferindo diretamente na qualidade da assistência. A baixa capacitação profissional gera insegurança sobre os deveres de notificação e denúncia de casos suspeitos. Conclusão: É necessário manter os profissionais capacitados e aptos para atuar em casos de violência contra a mulher, promovendo o bem-estar da vítima, família e comunidade.

Palavras-chave: violência contra a mulher, atenção primária à saúde, profissionais de saúde
\end{abstract}

\begin{abstract}
Introduction: The violence against women causes an increase in the demand for health services that are fundamental to recognize the situation of violence through clinical analysis and reception. Objective: To analyze the knowledge of health professionals in primary care about the violence against women, and to verify their conduct during care. Methods: Cross-sectional quantitative study, carried out with thirty health professionals of a basic health unit, through a questionnaire composed of questions of personal and professional data and understanding about domestic violence. Results and discussion: Most interviewees deny previous knowledge about domestic violence, which directly interferes with the quality of care. Low professional training creates uncertainty about their notification duties and reporting suspected cases. Conclusion: It is necessary to keep professionals trained and able to act in cases of violence against women, promoting the well-being of the victim, family, and community.
\end{abstract}

Keywords: violence against woman, primary health care, health professionals

\section{Resumen}

Introducción: La violencia contra la mujer genera un aumento en la demanda de los servicios de salud que son fundamentales en el reconocimiento de las situaciones de violencia a través del análisis clínico y la acogida. Objetivo: Analizar el conocimiento de los profesionales de la salud en atención primaria ante la violencia contra las mujeres y verificar su conducta durante la atención. Métodos: Estudio transversal cuantitativo, desarrollado con treinta profesionales de salud de una unidad básica de salud, a través de un cuestionario compuesto por preguntas de datos personales, profesionales y conocimiento sobre la violencia doméstica. Resultados y discusión: La mayoría de los entrevistados niega conocimiento previo sobre violencia doméstica, lo que interfiere directamente en la calidad de la asistencia. La baja capacitación profesional genera inseguridad frente a sus deberes de notificación y denuncia de casos sospechosos. Conclusión: Es necesario mantener profesionales capacitados y capaces de actuar en casos de violencia contra la mujer, promoviendo el bienestar de la víctima, la familia y la comunidad.

Palabras clave: violencia contra la mujer, atención primaria a la salud, profesionales de la salud

\footnotetext{
${ }^{1}$ Endereço para contato: Rua Acre, 91, Bairro Jardim Imá, CEP 79102-140, Campo Grande, Mato Grosso do Sul, Brasil. Telefone: (67) 99179-3456. E-mail: rafaelafusquine@hotmail.com
} 


\section{Introdução}

A violência contra a mulher é definida como um conjunto de todos os atos de violência física, psicoemocional, sexual e desrespeito aos direitos em qualquer momento da vida pública ou particular dessa mulher, podendo ser cometida por qualquer pessoa que esteja, ou não, inserida nesse contexto social (Pessoa, 2002). Entre as diversas situações de violência em que a mulher se encontra, a violência doméstica é destacada, sendo descrita como todas as formas de violência e comportamentos violentos praticadas no âmbito familiar, cometidas por um membro da família ou pessoa que habite ou tenha habitado o mesmo domicílio (Diniz, Lopes, Gesteira, Alves, \& Gomes, 2003).

Os fatores geradores da situação de violência são divididos em precipitantes e condicionantes; entre os fatores precipitantes, estão: o consumo de álcool e drogas; o estresse e cansaço mental que são motivações do descontrole psicoemocional, os quais podem desencadear agressividade. Os fatores condicionantes se manifestam pelas desigualdades enfrentadas pelas mulheres na sociedade em comparação com os homens, como as de ordem econômicas, educacionais, profissionais, entre outras (Gregori, 1992).

A violência doméstica está inserida em diferentes culturas e sociedades. Segundo um estudo realizado com mulheres usuárias do serviço primário de saúde, 44,4\% havia passado por experiência de violência doméstica envolvendo agressão física, sendo 34,1\% partindo de companheiros ou familiares. Ainda, neste estudo, foi observada uma ocorrência de violência sexual em $11,5 \%$ das mulheres, sendo, deste total, 7,1\% de casos praticados por companheiros ou familiares (Schraiber, D’Oliveira, França Junior, \& Pinho, 2002).

Diante do aumento da visibilidade da violência contra a mulher em nível nacional e mundial, leis foram estabelecidas para a proteção dos direitos das mulheres, dando origem a políticas públicas necessárias para a assistência às mulheres vítimas de violência. Entre essas intervenções para a melhora na qualidade da assistência à mulher, ressaltam-se a criação das delegacias de defesa à mulher e a criação da Lei Maria da Penha, n. 11.340/2006, que trata da punição de agressores em âmbito doméstico ou familiar (Brasil, 2006). Além disso, vale ressaltar que a Política Nacional de Atenção Integral à Mulher inclui o atendimento às mulheres em situação de violência, implementando diretrizes ao acompanhamento das muIheres em situações de vulnerabilidade (Brasil, 2004).

Segundo dados levantados pela Comissão Interamericana de Direitos Humanos (CIDH), foram computados ao menos 126 casos de feminicídio no mês de janeiro do ano de 2019. Esses dados são referentes a casos registrados em 159 cidades distribuídas por todo o Brasil. Concomitante a essa publicação, a CIDH adiciona que mantém preocupações motivadas pela prevalência alarmante dos índices de feminicídio no Brasil (Santana, 2019).

Em adição aos dados expostos anteriormente, a Comissão Econômica para a América Latina e o Caribe (CEPAL), das Organizações Unidas, expõe que $40 \%$ de todos os casos de feminicídios registrados na América Latina e no Caribe ocorrem no Brasil (CEPAL, 2015). Essa informação concorda com os dados de 2019 do Atlas da Violência, que evidenciou que, em dez anos (entre 2007 e 2017), houve um aumento gradativo no número de casos de feminicídios no Brasil, sendo os maiores números computados em 2017, nos estados de São Paulo (495), Bahia (487) e Rio de Janeiro (401), respectivamente, o que evidencia um alto índice de vulnerabilidade das mulheres no país (Instituto de Pesquisa e Economia Aplicada [IPEA] , 2019). 
A violência contra a mulher é tida como problema de saúde pública, por atingir mulheres de diversas esferas sociais e por esta situação traduzir, muitas vezes, problemas de saúde capazes de interferir na qualidade de vida dessas mulheres. A violência, em geral, acarreta problemas psicossociais e mentais, envolvendo o aumento de taxas de suicídio, uso abusivo de álcool e drogas, cefaleias recorrentes e distúrbios gastrointestinais, entre outros ((Schraiber et. al, 2002) . A violência sexual, além da possibilidade de ocorrência de todos os outros acarretamentos anteriormente citados, interfere na saúde reprodutiva da vítima, ocasionando dores pélvicas crônicas em conjunto com doenças pélvicas inflamatórias, infecções sexualmente transmissíveis (IST) curáveis e não curáveis e gravidez indesejada (Fontana \& Santos, 2001).

Com isso, nota-se a maior procura das mulheres vítimas de violência nos serviços de saúde, que, mesmo que indiretamente, têm papel importante no reconhecimento das situações de violência por meio da análise clínica e no acolhimento das mulheres antes do agravamento da situação (Kronbauer \& Meneghel, 2005).

Sabe-se que a Atenção Básica de Saúde é umas das principais portas de entrada para as mulheres que vivem em situação de violência (Pimenta, 2011), logo, é importante que haja uma hábil e coerente estratégia de acolhimento emergencial que avalie essa paciente como um ser complexo e multifacetado. É importante ressaltar que, por conta dos princípios organizativos da Atenção Primária à Saúde (APS), como territorialização, integralidade, assistência continuada, rede de serviços de saúde e outras, existe uma maior possibilidade de promoção, prévia identificação e prevenção de casos de violência contra a mulher (Costa, 2011).

Atualmente, a APS assiste grande porção dos casos de violência doméstica contra crianças e mulheres, ainda que de forma indireta e sem serem apresentados como tal. Esse cenário é presente no cotidiano dos serviços e ainda gera uma demanda reiterada e, ainda, com baixa resolutividade (Waisefisz, 2015).

Ao oferecer cobertura e vínculo com as mulheres atendidas na unidade de saúde, os profissionais se fazem essenciais na detecção da ocorrência da violência em âmbito familiar (Schraiber et al., 2002). Porém, nem sempre a unidade de saúde oferece uma intervenção satisfatória ao problema, podendo esse se diluir entre outros agravos, sem ter ressaltado a etiologia do estado clínico da vítima. Essa invisibilidade é consequência da limitação do olhar aos sintomas e doença, sem contar com instrumentos capazes de identificar o causador (Silva, 2003), impossibilitando um reconhecimento da situação e possibilitando uma quebra de vínculo da usuária com o serviço de saúde, além da contribuição com a perpetuação do ciclo da violência (Santos, 2003).

Segundo estudiosos, um dos principais problemas na falha da identificação da violência contra a mulher é o seguimento do modelo biomédico, em que os profissionais se atentam aos sinais e sintomas delineados por uma possível doença; assim, é necessário que se desvinculem das condutas hospitalocêntricas, geralmente focadas em problemas clínicos. Além desse fator, o baixo número de capacitações expõe uma dificuldade ao abordar assuntos vistos como delicados e íntimos (Duarte et al., 2019).

Sendo assim, este estudo objetiva analisar o conhecimento dos profissionais de saúde da atenção básica ante a violência contra a mulher e verificar suas condutas durante o atendimento. 


\section{Método}

Trata-se de um estudo quantitativo transversal, desenvolvido em uma Unidade Básica de Saúde da Família localizada no município de Campo Grande, Mato Grosso do Sul, Brasil, o qual faz parte de um trabalho do PIBIC em andamento que teve a aprovação do Comitê de Ética em Pesquisa da Universidade Católica Dom Bosco, Parecer n. 2.049.305.

O estudo contou com a participação de 30 profissionais de saúde da unidade de saúde em estudo, de escolaridade de ensino médio, superior e técnico, sendo agentes comunitários de saúde e técnicos em enfermagem, médicos, enfermeiros, fisioterapeuta, assistente social e odontólogo atuantes na unidade de saúde, com idades entre 22 e 57 anos.

Os dados foram coletados no mês de julho de 2018, por meio de um questionário composto por quatro etapas: 1) dados pessoais e de formação; 2) dados relacionados ao conhecimento do profissional ante o atendimento recomendado pelos órgãos competentes; 3 ) dados relacionados ao conhecimento profissional sobre o que é a violência contra a mulher e suas vertentes; 4 ) dados referentes à percepção sobre a violência contra a mulher num ponto de vista pessoal. A distribuição dos dados foi realizada por meio de tabulação Excel ${ }^{\circledR}$, logo, eles foram analisados e confrontados com artigos científicos sobre a temática já publicados.

\section{Resultados}

Da totalidade de 30 profissionais de saúde entrevistados, 73,33\% ( $n=22)$ eram do sexo feminino, tendo uma média de idade de 35,1 . Entre as categorias profissionais, $10 \%(n=3)$ eram enfermeiros; $6,66 \%(n=2)$ eram médicos; $13,33 \%(n=4)$ eram técnicos em enfermagem; $56,66 \%(n=17)$, a maioria, agentes comunitários (ACS); e 3,33\% ( $n=1)$, a minoria, envolviam fisioterapeuta, odontólogo, psicólogo e assistente social.

Quanto ao histórico de formação, 50\% ( $n=15)$ formaram-se em instituição técnica ou de ensino superior pública, e outros $50 \%(n=15)$, em instituições privadas. Mais da metade (60\% e $n=18$ ) relatam não terem tido conteúdo sobre como lidar com situações de violência dentro da instituição de formação, sendo a maioria desse montante proveniente de universidades públicas (66,6\% e $n=12$ ). No âmbito de atuação profissional, 56,66\% ( $n=17)$ relataram não terem capacitação profissional oferecida pela instituição atuante sobre como lidar com situações de violência doméstica, e apenas $20 \%(n=6)$ relataram ter realizado algum atendimento prévio à mulher vítima de violência.

Tabela 1

Descrição de informações pessoais e de trabalho dos profissionais de saúde entrevistados em julho de 2018, em uma unidade básica de saúde em Campo Grande, MS

\begin{tabular}{lcc}
\hline \multirow{2}{*}{ Variáveis } & \multicolumn{2}{c}{ Respostas } \\
\cline { 2 - 3 } & N & \% \\
\hline Sexo & & \\
$\quad$ Feminino & 22 & 73,33 \\
$\quad$ Masculino & 08 & 26,66 \\
\hline Idade & & \\
$\quad \leq 30$ anos & 11 & 36,67 \\
$31-39$ anos & 10 & 33,33 \\
$\quad \geq 40$ anos & 109 & 30,00 \\
\hline
\end{tabular}




\begin{tabular}{lcc}
\hline \multirow{2}{*}{ Variáveis } & \multicolumn{2}{c}{ Respostas } \\
\cline { 2 - 3 } & $\mathbf{N}$ & $\%$ \\
\hline Categorias profissionais & 17 & 56,66 \\
$\quad$ Agente Comunitário de Saúde & 01 & 3,33 \\
$\quad$ Assistente social & 03 & 10 \\
Enfermeiro & 01 & 3,33 \\
$\quad$ Fisioterapeuta & 02 & 6,66 \\
$\quad$ Médico & 01 & 3,33 \\
$\quad$ Odontólogo & 01 & 3,33 \\
$\quad$ Psicólogo & 04 & 13,33 \\
$\quad$ Técnico em Enfermagem & & \\
\hline Instituição & 15 & 50,00 \\
$\quad$ Privado & 15 & 50,00 \\
$\quad$ Público & & \\
\hline Obteve conteúdo sobre a conduta em situações de violência & & \\
doméstica? & 12 & 40,00 \\
$\quad$ Sim & 18 & 60,00 \\
$\quad$ Não & & \\
\hline Obteve capacitação para lidar com situações de violência doméstica & & \\
na instituição atuante? & 13 & 43,33 \\
$\quad$ Sim & 17 & 56,66 \\
$\quad$ Não & & \\
\hline Já realizou atendimento de vítimas de violência? & 06 & 20,00 \\
Sim & 24 & 80,00 \\
Não & &
\end{tabular}

No âmbito de conhecimento profissional ante as condutas ideais e recomendadas pelos órgãos de referência, 53,33\% ( $n=16)$ acreditam que a combinação de manejo clínico e o encaminhamento a outros profissionais são os procedimentos que devem ser tomados. A maioria dos entrevistados (93,33\% ou $n=28$ ) acredita que os profissionais condutores da consulta devem encorajar a mulher a sair da situação de violência sem confrontar seus pensamentos, e 96,66\% ( $n=29)$ acreditam que devem ser divulgados às vítimas informações, contatos e outros meios de atendimentos policiais durante a consulta. Apenas $23,33 \%(n=7)$ reconhecem a Casa da Mulher Brasileira, delegacia de apoio à mulher e a unidade de saúde como locais em que a mulher pode buscar auxílio.

Tabela 2

Descrição das percepções dos profissionais de saúde entrevistados sobre violência doméstica contra a mulher, em julho de 2018, em uma unidade básica de saúde em Campo Grande, MS

\begin{tabular}{|c|c|c|}
\hline \multirow{2}{*}{ Perguntas } & \multicolumn{2}{|c|}{ Respostas } \\
\hline & $\mathbf{N}$ & $\%$ \\
\hline \multicolumn{3}{|c|}{$\begin{array}{l}\text { Qual a conduta ideal dos profissionais de saúde em casos de violência } \\
\text { doméstica contra a mulher? }\end{array}$} \\
\hline Conduta clínica & 17 & 56,66 \\
\hline Encaminhamento a outros profissionais & 29 & 96,66 \\
\hline
\end{tabular}




\begin{tabular}{|c|c|c|}
\hline \multirow{2}{*}{ Perguntas } & \multicolumn{2}{|c|}{ Respostas } \\
\hline & $\mathbf{N}$ & $\%$ \\
\hline \multicolumn{3}{|c|}{$\begin{array}{l}\text { Você acredita que o profissional de saúde deve encorajar a mulher a } \\
\text { sair da situação de violência? }\end{array}$} \\
\hline Sim & 28 & 93,33 \\
\hline Não & 02 & 6,66 \\
\hline \multicolumn{3}{|c|}{$\begin{array}{l}\text { Os profissionais devem divulgar informações sobre atendimentos } \\
\text { policiais às mulheres em situação de violência? }\end{array}$} \\
\hline Sim & 29 & 96,66 \\
\hline Não & 01 & 3,33 \\
\hline \multicolumn{3}{|c|}{ Qual local você indicaria à mulher em situação de violência? } \\
\hline Qualquer delegacia & 13 & 43,33 \\
\hline Instituições especializadas & 27 & 90,00 \\
\hline Unidade de saúde & 12 & 40,00 \\
\hline \multicolumn{3}{|c|}{$\begin{array}{l}\text { O profissional de saúde deve denunciar casos de violência doméstica } \\
\text { contra a mulher? }\end{array}$} \\
\hline $\operatorname{sim}$ & 15 & 50,00 \\
\hline Não & 15 & 50,00 \\
\hline \multicolumn{3}{|c|}{$\begin{array}{l}\text { Você acredita que o profissional de saúde deve tratar de possíveis } \\
\text { lesões sem interferir na condição pessoal? }\end{array}$} \\
\hline Sim & 12 & 40,00 \\
\hline Não & 18 & 60,00 \\
\hline \multicolumn{3}{|c|}{$\begin{array}{l}\text { Você acredita que a agressão à mulher é um assunto de fórum íntimo } \\
\text { e privado? }\end{array}$} \\
\hline Sim & 08 & 26,66 \\
\hline Não & 22 & 73,33 \\
\hline
\end{tabular}

Entre os participantes da pesquisa, 50\% acreditam que o profissional de saúde não deve realizar a denúncia de suspeita de casos de violência doméstica, mesmo que a violência não seja confirmada, e a maior parte dos entrevistados $(60 \%$ ou $n=18)$ relata que os profissionais de saúde devem tratar as lesões sem interferir na condição pessoal, levando em conta que $26,6 \%(n=8)$ acreditam que a agressão domiciliar é um assunto de fórum íntimo e privado.

Quase a totalidade dos profissionais questionados $(96,66 \%$ ou $n=29)$ demonstrou saber que, na maioria dos casos, a violência doméstica contra a mulher é cometida por pessoas conhecidas e presentes na rotina das mulheres. Quanto ao relato da violência, 83,33\% ( $n=25)$ dos entrevistados reconhecem que a violência contra a mulher não é relatada ao profissional de saúde.

A maioria dos profissionais $60 \%(n=18)$ acredita que a lesão corporal não está presente na totalidade das agressões, tendo em vista que $100 \%(n=30)$ dos entrevistados acreditam que a violência contra a mulher engloba, além da agressão física, a agressão verbal e a psicoemocional. 
Tabela 3

Continuação das percepções dos profissionais de saúde entrevistados sobre violência doméstica contra a mulher, em julho de 2018, em uma unidade básica de saúde em Campo Grande, MS

\begin{tabular}{|c|c|c|}
\hline \multirow{2}{*}{ Perguntas } & \multicolumn{2}{|c|}{ Respostas } \\
\hline & $\mathbf{N}$ & $\%$ \\
\hline \multicolumn{3}{|c|}{$\begin{array}{l}\text { Você considera que violência contra a mulher, em sua maioria, é } \\
\text { causada por pessoas conhecidas? }\end{array}$} \\
\hline Sim & 29 & 96,66 \\
\hline Não & 01 & 3,33 \\
\hline \multicolumn{3}{|c|}{$\begin{array}{l}\text { Você considera violência contra a mulher: gritos, xingamentos, } \\
\text { persuasão, sexo forçado, tapas, puxões de cabelos, impedimento } \\
\text { de trabalhar, impedimento de pintar o cabelo ou usar decote? }\end{array}$} \\
\hline Sim & 28 & 93,33 \\
\hline Não & 02 & 6,66 \\
\hline \multicolumn{3}{|c|}{$\begin{array}{l}\text { Você acredita que a violência é relatada, na maioria das vezes, ao } \\
\text { profissional de saúde pela vítima durante a consulta? }\end{array}$} \\
\hline $\operatorname{Sim}$ & 05 & 16,66 \\
\hline Não & 25 & 43,33 \\
\hline \multicolumn{3}{|c|}{$\begin{array}{l}\text { Você acredita que a lesão corporal está presente na maioria das } \\
\text { agressões contra a mulher? }\end{array}$} \\
\hline $\operatorname{sim}$ & 18 & 60,00 \\
\hline Não & 12 & 40,00 \\
\hline \multicolumn{3}{|c|}{ Você acredita que o agressor deve ser penalizado judicialmente? } \\
\hline Sim & 30 & 100,00 \\
\hline Não & 00 & 0,00 \\
\hline \multicolumn{3}{|c|}{$\begin{array}{l}\text { Assinale o que você acredita que possa ser a causa de uma situação } \\
\text { de violência doméstica contra a mulher }\end{array}$} \\
\hline Abuso de álcool & 27 & 90,00 \\
\hline Abuso de drogas & 26 & 86,66 \\
\hline Transtorno psicológico & 23 & 76,66 \\
\hline Cansaço mental & 13 & 43,33 \\
\hline Descontentamento do parceiro ante a relação & 10 & 33,33 \\
\hline Responsabilidade da parceira & 04 & 13,33 \\
\hline Outros & 03 & 10,00 \\
\hline
\end{tabular}

A totalidade de 100\% ( $n=30)$ dos entrevistados acredita que os agressores devem ser penalizados judicialmente. Entre as causas apontadas para a ocorrência da violência doméstica, foram citados o abuso de álcool ( $90 \%$ e $n=27)$, abuso de drogas $(86,66 \%$ e $n=26)$, transtorno psicológico (76,66\% e $n=23)$, cansaço mental (43,33\% e $n=13)$, descontentamento do parceiro $(33,33 \%$ e $n=10)$ e responsabilidade da agredida $(13,33 \%$ e $n=4)$. Ressalta-se a presença de outras opiniões, como a interferência do modo de criação, o machismo culturalmente presente (10\% e $n=3)$.

Vale destacar que, ao analisar os dados em combinação, foi possível perceber que, entre as pessoas que tinham um conhecimento prévio sobre violência contra a mulher provindo da formação, capacitação profissional ou experiência de atendimento prévio (53,3\% e $n=16)$, existiram ainda erros sobre as condutas corretas do profissional durante o atendimento $(37,5 \%$ e $n=6)$ e locais que fornecem atendimento à mulher vítima de violência $(75 \%$ 
e $n=12)$. Além disso, ainda entre essa amostra, 43,7\% ( $n=7)$ não tinham conhecimento sobre a proporção da lesão física diante de outros tipos de violência contra a mulher. Logo, nessa análise, não é possível relacionar o conhecimento prévio com a ideal conduta esperada.

Evidencia-se também que, entre o montante que declarou acreditar que a violência contra a mulher é de fórum íntimo e privado $(26,6 \%$ e $n=8)$, a maioria $(62,5 \%$ e $n=5)$ denunciaria e $100 \%$ ( $n=8$ ) ofereceria dados e informações sobre atendimento policial durante um atendimento profissional. Assim, é possível evidenciar que a concepção pessoal do atuante não interfere em sua conduta profissional.

\section{Discussão}

A especialização ou a apresentação do tema abordado durante a formação acrescenta um conhecimento adicional necessário e se faz presente, principalmente, em instituições onde há especializações voltadas ao feminismo, à desconstrução do machismo em sociedade, à obstetrícia e ginecologia (Gutsmanis, Beynon, Tutty, Wathen, \& MacMillan, 2019). A situação de desconhecimento empírico ante a ocorrência de violência doméstica é evidenciada quando se aborda a escassez de vínculo entre unidade de atenção primária e usuário do sistema de saúde, tendo em vista que, muitas vezes, o profissional não é capaz de enxergar a existência do problema. Uma articulação de atenção primária à saúde juntamente da equipe de estratégia de saúde da família intensifica o vínculo entre população e profissionais de saúde, integrando-os nas rotinas do serviço e das famílias, abrindo as portas para a detecção de problemas diversificados (Ferreira Neto \& Kind, 2011).

O combate à violência doméstica contra a mulher necessita de uma equipe de apoio que vai além do serviço de saúde para que os problemas sejam identificados e resolvidos, porém, em geral, os serviços de saúde carecem de profissionais capacitados na avaliação e no reconhecimento dos sinais de violência (Brasil, 1999). Segundo estudos, quando a mulher é identificada em uma situação de risco, ela deve ser estimulada a realizar outros tipos de atendimentos, como psicoterápicos; além disso, a mulher deverá receber assistência aos danos físicos e orientações para conclusão do registro policial (Rotania et al., 2003).

Autores (Schraiber et al., 2003) indicam que os serviços de saúde podem ser utilizados como facilitadores da identificação dos eventos violentos por conta da somatização dos problemas físicos decorrentes da situação de violência, os quais são capazes de originar depressões e outros casos mórbidos; segundo estudos, em 38\% dos casos de violência doméstica, o suicídio é considerado como alternativa de escape (Adeodato, Carvalho, Siqueira, \& Souza, 2005).

Em estudos realizados com profissionais de saúde, houve relatos de dificuldades ante a denúncia, apesar de o profissional ser responsável pela saúde da comunidade e ter o dever de notificar casos de vulnerabilidade. Também, muitas vezes, a denúncia anônima é vista como estratégia para outros tipos de relatos (Porto, Bispo Junior, \& Lima, 2014). Há uma divergência entre a notificação dos casos e a denúncia propriamente dita. Apresentar-se favorável ante a notificação faz com que haja melhora na visibilidade da violência contra a mulher. A não notificação dos casos dificulta o mapeamento em benefício do combate à violência. Pesquisas informam que, apesar de os profissionais de saúde serem obrigados a notificar casos de violência, eles se sentem inúteis diante das situações. Muitos deles veem 
a notificação como uma denúncia e alegam dúvidas do desdobramento que a notificação acarreta para os serviços de saúde, gerando inseguranças e medo ao se envolver com o caso (Adeodato et al., 2005).

Os homens vivenciam a maior probabilidade de serem vítimas de violência com pessoas estranhas e pouco conhecidas, enquanto as mulheres, ao contrário, apresentam maior probabilidade de serem vítimas de suas próprias famílias e parceiros. De 40\% a 70\% dos feminicídios são cometidos por parceiros íntimos (Day et al., 2003). Geralmente, a violência contra a mulher é cometida por pessoas que estejam numa relação de poder com a vítima. A maioria dos casos de violência ocorre no âmbito domiciliar e, em adição de preceitos machistas, envolve agressores que assumem a função de pai, padrasto ou cônjuge (Brasil, 1941).

Pesquisadores acreditam haver um pacto entre usuárias vítimas de violência, que, por sua vez, não relatam a agressão, e os profissionais de saúde também não perguntam (Schraiber et al., 2003). Chamada de "invisibilidade", essa situação tem sido rompida pelo sucesso do reconhecimento gradiente da violência contra a mulher; tal violência, para ser interrompida, depende fortemente da abordagem dos profissionais de saúde (D’Oliveira \& Schraiber, 1999).

A identificação dos casos de violência depende de uma intensa capacitação do profissional de saúde, a qual envolve, além de bagagem teórica e científica, sensibilidade, habilidade e disponibilidade para atuar na escuta ativa. Como estratégia de abordagem, o Ministério da Saúde recomenda a adoção de perguntas diretas durante o atendimento à mulher em situação de violência (Schraiber et al., 2003).

Estudos indicam que a incidência de violência doméstica é maior em agressores que convivem com o abuso de substâncias psicoativas independentemente da sociedade, cultura e grupos sociais (D’Oliveira \& Schraiber, 1999). Em uma pesquisa realizada no México, foi observada uma prevalência de $42 \%$ de violência sexual, $40 \%$ de violência física e $38 \%$ de violência emocional, em que o abuso de álcool e droga sintetizou os fatores causais das três dimensões estudadas (Alvarado-Zaldivar, Moysém, Estrada-Martinez, \& Terrones-Gonzales, 2002).

\section{Conclusão}

A violência contra a mulher é uma realidade da atenção básica e os profissionais de saúde são mais próximos da população, sendo capazes de obter informações sobre a rede de usuário e estabelecer vínculo de confiança entre as mulheres e o serviço de saúde. O serviço de saúde pode ser identificado como um instrumento de detecção de situações de violência, mas, para que isso seja possível, é necessário que haja a criação de um ambiente humanizado que proporcione acolhimento com respeito e sem julgamentos, de uma forma constante, não só em situações de vulnerabilidade perceptível.

Foi possível identificar, neste estudo, que fragilidades na atenção às situações de violência assistidas na atenção primária em saúde provêm da escassez de informação durante o processo de formação profissional e de aspectos conceituais, inclusive, a visão biomédica ainda presente. Porém a falta de conhecimento ou contato prévio com casos de violência contra a mulher não isenta o profissional da importância de conhecer e saber atuar diante dessa situação. 
Ficou claro que a concepção pessoal da amostra estudada não foi suficiente para interferir na conduta do profissional, uma vez que suas próprias opiniões divergiram das condutas assinaladas para um possível atendimento. Com essa informação, pode-se concluir que o atendimento profissional a essas vítimas é geralmente encaminhado conforme o conhecimento adquirido numa capacitação ou numa situação empírica prévia.

Logo, conclui-se que é importante em demasia manter os profissionais conceituados e treinados, de forma rotineira e com qualidade, para que haja uma preparação na atuação, sem que esta seja prejudicial para a vítima, família e comunidade.

\section{Referências}

Adeodato, V. G., Carvalho, R. R., Siqueira, V. R., \& Souza, F. G. M. (2005). Qualidade de vida e depressão em mulheres vítimas de seus parceiros. Revista Saúde Pública, 39(1), 108-113. doi:http://dx.doi.org/10.1590/S0034-89102005000100014

Alvarado-Zaldivar, G., Moysén, J. S., Estrada-Martínez, S., \& Terrones-González, A. (2002).

Prevalencia de violência doméstica enlaciudad de Durango. Salud Publica de Mexic, 40(1), 481-486.

Atlas da violência. (2019). Rio de Janeiro: Ipea, FBSP.

Brasil. (1941). Lei n. 3.688, de 3 de outubro de 1941. Lei das Contravenções Penais. Recuperado de http://www.planalto.gov.br/ccivil_03/Decreto-Lei/Del3688.htm

Brasil. (1999). Ministério da Saúde. Prevenção e tratamento dos agravos resultantes da violência sexual contra mulheres e adolescentes. Brasília-DF: Ministério da Saúde.

Brasil. (2004). Ministério da Saúde. Política Nacional da Atenção Integral à Saúde da Mulher: princípios e diretrizes. Secretaria da Atenção à Saúde. Departamento de Ações Programáticas Estratégicas. Brasília-DF: Ministério da Saúde.

Brasil. (2006). Lei 11.340, de 7 de agosto de 2006. Cria mecanismos para coibir a violência doméstica e familiar contra a mulher, nos termos do 8o do art. 226 da Constituição Federal. Diário Oficial da União, 08 de Agosto de 2006. Disponível em: http://www.planalto.gov. br/ccivil_03/_ato2004-2006/2006/lei/l11340.htm

Comisión Económica para América Latina y el Caribe. (2015). Informe anual 2013- 2014. El enfrentamiento de la violencia contra las mujeres en América Latina y el Caribe (LC/G.2626), Santiago de Chile: CEPAL.

Costa, L. H. R. (2011) Estendendo o Fio de Ariadne: Sexualidade feminina e a interseção com o cuidado nos discursos de enfermeiras (Tese, Universidade Federal da Bahia).

Day, V. P., Telles, L. E. B., Zoratto, P. H., Azambuja, M. R. F., Machado, D. A., Silveira, M. B., ... Blamk, R. (2003). Violência doméstica e suas diferentes manifestações. Revista de Psiquiatria do Rio Grande do Sul, 25(1), 9-21.

Diniz, N. M. F., Lopes, R. L. M., Gesteira, M. A., Alves, S. L. B., Gomes, N. P. Violência conjugal: Vivências expressas em discursos masculinos. Revista Escola de Enfermagem, 37(2), 81-88.

D’Oliveira, A. F. P. L., \& Schraiber, L. B. (1999). Violência de gênero como uma questão de saúde: A importância da formação dos profissionais. Jornal da Rede Feminista de Saúde, 19, 3-4.

Duarte, B. A. R., Junqueira, M. A. B., \& Giuliani, C. D. (2019). Vítimas de violência: Atendimento dos profissionais de enfermagem em Atenção Primária. Revista REFACS. 7(3), 401-411. 
Ferreira Neto, J. L., Kind, L. (2011). Promoção da saúde e práticas grupais na Estratégia de Saúde da Família. São Paulo: Editora Hucitec.

Fontana, M., Santos, S. F. (2001). Violência contra a mulher. São Paulo: Rede Nacional Feminista de Saúde e Direitos Reprodutivos (organizador). [Saúde da mulher e direitos reprodutivos: dossiês].

Gregori, M. F. (1992). Cenas e queixas: Um estudo sobre mulheres, relações violentas e a prática feminista. São Paulo: ANPOCS.

Gutmanis, I., Beynon, C., Tutty, L., Wathen, C. N., \& MacMillan, H. L. (2007). Factors influencing identification of and response to intimate partner violence: A survey of physicians and nurses. BMC Public Health, 7(12), 1-11..

Kronbauer, J. F. D., \& Meneghel, S. N. (2005). Perfil da violência de gênero perpetrada por companheiro. Revista Saúde Pública, 39(5), 695-701. doi: http://dx.doi.org/10.1590/ S0034-89102005000500001

Pessoa, S. B. (2002). Mulheres em situação de violência: Guia de serviços. São Paulo, SP: Coletivos Feminista Sexualidade e Saúde; Universidade de São Paulo.

Pimenta, J. C. (2011). Violência contra mulher: Um desafio para a atenção básica à saúde (Monografia, Universidade Federal de Minas Gerais, Governador Valadares, NESCON). Recuperado de https://www.nescon.medicina.ufmg.br/biblioteca/registro/ Violencia_contra_mulher_um_desafio_para_a_atencao_basica_a_saude/459

Porto, R. T. S., Bispo Junior, J. P., \& Lima, E. C. (2014). Violência doméstica e sexual no âmbito da Estratégia de Saúde da Família: Atuação profissional e barreiras para o enfrentamento. Physis, 24(3), 787-807.

Rotania, A. A., Dias, M. I. V., Ventura, K. S., Wolf, L. R., Belz, L. R., \&Tyrrell, M. A. R. (2003). Violência contra a mulher: O perigo mora da porta para dentro. Escola Anna Nery Revista de Enfermagem, 7(1), 11-125.

Santana, G. R. (2019). Feminicídio no Brasil em 2019: Reflexões sobre a notícia 24/19 da Comissão Interamericana de Direitos Humanos - CIDH. Revista de Gênero, Sexualidade e Direito, 5(1), 40-57.

Santos, L. L. (2003). A visibilidade da violência de gênero em dois serviços de assistência primária à saúde (Dissertação, Faculdade de Medicina de Ribeirão Preto, Ribeirão Preto, Universidade de São Paulo).

Schraiber, L. B., D’Oliveira, A. F. P. L., França Junior, I., \& Pinho, A. A. (2002). Violência contra a mulher: Estudo em uma unidade de atenção primária à saúde. Revista Escola de Enfermagem, 36(4), 470-477.

Schraiber, L. B., D’Oliveira, A. F. P. L., Hanada, H., Figueiredo, W., Couto, M., Kiss, L., ... Pinho, A. (2003) Violência vivida: A dor que não tem nome. Interface (Botucatu), 7(12), 41-54.

Silva, I. V. (2003). Violência contra mulheres: A experiência de usuárias de um serviço de urgência e emergência de Salvador, Bahia, Brasil. Cadernos de Saúde Pública, 19(2), 263-272.

Waiselfisz, J. J. (2015). Mapa da violência 2015: Homicídio de mulheres no Brasil. Brasília-DF: quem publicou.

Recebido em: 27/05/2019 última revisão: 09/03/2020

Aceite final: 27/03/2020 


\section{Sobre as autoras:}

Rafaela Serrano Fusquine: Aluna do Programa Institucional de Bolsas de Iniciação Científica (PIBIC) no âmbito de Atenção à Saúde da Mulher Vítima de Violência Sexual. Discente do curso de graduação em Enfermagem da Universidade Católica Dom Bosco (UCDB). E-mail: rafaelafusquine@ hotmail.com, Orcid: https://orcid.org/0000-0003-4286-0183

Yasmin Alves de Souza: Aluna do Programa Institucional de Bolsas de Iniciação Científica (PIBIC) no âmbito de Atenção à Saúde da Mulher no Puerpério. Discente do curso de Atenção à Saúde da Mulher Vítima de Violência Sexual. Discente do curso de graduação em Enfermagem da Universidade Católica Dom Bosco (UCDB), Brasil.E-mail: yasminmin_@hotmail.com, Orcid: https://orcid.org/0000-0002-8254-1307

Aucely Corrêa Fernandes Chagas: Doutora em Saúde e Desenvolvimento na Região Centro-Oeste pela Universidade Federal de Mato Grosso do Sul (UFMS). Docente da Universidade Católica Dom Bosco (UCDB). Professora e orientadora no curso de Pós-Graduação Lato Sensu a distância pelo convênio UCDB/Portal da Educação e do Curso de Graduação em Enfermagem. E-mail: aucely@ucdb.br, Orcid: https://orcid.org/0000-0002-6682-346X 